\title{
Computing the Canonical Representation of Constructible Sets
}

\author{
Josep M. Brunat · Antonio Montes
}

Received: 29 June 2015 / Revised: 14 January 2016 / Accepted: 23 February 2016

(C) Springer International Publishing 2016

\begin{abstract}
Constructible sets are needed in many algorithms of Computer Algebra, particularly in the Gröbner Cover and other algorithms for parametric polynomial systems. In this paper we review the canonical form of constructible sets and give algorithms for computing it.
\end{abstract}

Keywords Constructible sets · Locally closed sets - Canonical representation · Parametric polynomial system . Gröbner Cover · Comprehensive Gröbner system

Mathematics Subject Classification $13 \mathrm{P} 10 \cdot 68 \mathrm{~T} 15$

\section{Introduction}

In the basic paper defining the Gröbner Cover [16] for discussing parametric polynomial systems of equations, we introduced algorithms that have been improved since then. We used our own algorithm BUILDTREE for computing the initial Comprehensive Gröbner System (CGS), needed for the Gröbner Cover, now substituted in the Singular [7] library "grobcov.lib" by the more efficient Kapur-Sun-Wang algorithm [11]. The algorithm GROBCOV used specially simple locally closed sets, whose union is certified to be also locally closed by Wibmer's theorem [17] (algorithm LCUNION).

The Gröbner Cover is used in [15] for the automatic deduction of geometric theorems. It is also essential for computing geometrical loci and defining a taxonomy of the components of loci in [1], as well as for envelopes. In general in these tasks, the representation of locally closed sets, i.e. difference of varieties, is sufficient. But for more general applications, where Wibmer's theorem [17] is not applicable, the union of locally closed sets is not always locally closed. This is the reason for reviewing here the canonical representation of constructible sets giving algorithms to compute it, as well as to use the new algorithms inside the library for computing higher dimensional

Partially supported by MTM2011-24097 and Gen. Cat. DGR 2014SGR46.

J. M. Brunat · A. Montes $(\varangle)$

Universitat Politècnica de Catalunya, Barcelona, Spain

e-mail: antonio.montes@upc.edu

J. M. Brunat

e-mail: Josep.M.Brunat@upc.edu 
geometrical loci's. As shown in Example 2, the algorithm for computing the canonical form of constructible sets can be very useful in an alternative construction of the Gröbner Cover.

Canonical form of constructible sets were already introduced by [3], in the context of general topology. More recently, [10] have given a description of invariant sequences for constructible sets in Zariski topology. The object of this paper is, taken this last description as starting point, to give formulas and algorithms for computing effectively the canonical form of constructible sets.

In Sect. 2, we give the two canonical representations of locally closed sets and an algorithm PCREP for computing them, that is central for our purposes. In Sect. 3, we recall the canonical structure of constructible sets introduced by [10], complementing it with dimension characteristics and an effective formula. This formula allows us to give an algorithm in Sect. 4 to build the canonical representation of constructible sets, using the CREP for locally closed sets. In Sect. 4 we also propose an acceleration method. Finally in Sect. 5 clarifying examples are given. In particular an example shows why the new algorithms are promising for an alternative approach for building the Gröbner Cover.

Some remarks about notation. All along the paper we shall use the notations $\subseteq$ and $\subset$ to represent inclusion and strict inclusion, respectively. If $r \geq 1$ is an integer the symbol $[r]$ means the set $[r]=\{i \in \mathbb{N}: 1 \leq i \leq r\}$. For a set $S \subseteq \mathbb{C}^{n}$, the complementary set $\mathbb{C}^{n} \backslash S$ of $S$ is denoted $S^{c}$. Finally $A \uplus B$ means disjoint reunion, that is, $A \cup B$ with the additional information that $A \cap B=\emptyset$.

\section{Canonical Representations of Locally Closed Sets}

Consider the ring $\mathbb{Q}[\mathbf{x}]=\mathbb{Q}\left[x_{1}, \ldots, x_{n}\right]$ of polynomials in $n$ indeterminates $x_{1}, \ldots, x_{n}$ with rational coefficients. If $N \subseteq \mathbb{Q}[\mathbf{x}]$, the variety of $N$ is the set

$\mathbf{V}(N)=\left\{\mathbf{u} \in \mathbb{C}^{n}: g(\mathbf{u})=0\right.$ for all $\left.g \in N\right\}$.

Let $\mathfrak{a}=\operatorname{RAD}(\langle N\rangle)$. Then $\mathbf{V}(N)=\mathbf{V}(\langle N\rangle)=\mathbf{V}(\mathfrak{a})$. The ideal $\mathfrak{a}$ is called the ideal of the variety $\mathbf{V}(N)$, and is denoted $\mathfrak{a}=\mathbf{I}(\mathbf{V}(N))$. If $S \subseteq \mathbb{C}^{n}$, the closure of $S$ is the smallest variety containing $S$, and is denoted $\bar{S}$. The ideal of $S$, denoted $\mathbf{I}(S)$, is defined by $\mathbf{I}(S)=\mathbf{I}(\bar{S})$. By the Nullstellensatz, there is a one-to-one correspondence between varieties $V$ and radical ideals $\mathfrak{a}$. For a radical ideal $\mathfrak{a}$ and a variety $V$, both $\mathbf{I}(\mathbf{V}(\mathfrak{a}))=\mathfrak{a}$ and $\mathbf{V}(\mathbf{I}(V))=V$ hold.

By taking varieties as closed sets, we have a topology in $\mathbb{C}^{n}$ called the $\mathbb{Q}$-Zariski topology of $\mathbb{C}^{n}$. For concepts about varieties and the $\mathbb{Q}$-Zariski topology of $\mathbb{C}^{n}$ not defined here (such as irreducible varieties, irreducible components, dimension of a variety, etc.), we refer to [2,5].

A set $S \subseteq \mathbb{C}^{n}$ is locally closed if it is the intersection of an open set and a closed set.

Remark 2.1 The concept of locally closed set admits different but equivalent definitions. Indeed, the following conditions are easily shown to be equivalent:

(a) The set $S$ is locally closed;

(b) the set $S$ is the difference of two closed sets;

(c) the set $S$ is open in the closure $\bar{S}$ of $S$;

(d) the set $\bar{S} \backslash S$ is closed.

Let $S$ be an open (resp. closed) set. As $\mathbb{C}^{n}$ is closed (resp. open), then $S=S \cap \mathbb{C}^{n}$ is a locally closed set. Thus, open sets and closed sets are locally closed.

We introduce now the canonical C-representation of a locally closed set $S$. Let $S$ be a locally closed set. As $\bar{S}$ and $\bar{S} \backslash S$ are closed, there exist radical ideals $\mathfrak{a}$ and $\mathfrak{b}$ such that

$\bar{S}=\mathbf{V}(\mathfrak{a}) \quad$ and $\quad \bar{S} \backslash S=\mathbf{V}(\mathfrak{b})$.

These ideals satisfy

$S=\bar{S} \backslash(\bar{S} \backslash S)=\mathbf{V}(\mathfrak{a}) \backslash \mathbf{V}(\mathfrak{b})$ 
Taking into account the one-to-one correspondence between radical ideals and varieties, the ideals $\mathfrak{a}=\mathbf{I}(\bar{S})$ and $\mathfrak{b}=\mathbf{I}(\bar{S} \backslash S)$ are uniquely determined by $S$. The pair $\operatorname{CREP}(S)=[\mathfrak{a}, \mathfrak{b}]$ is called the C-canonical representation of the locally closed set $S$. It is canonical in the sense that it does not depend on how the locally closed set $S$ is given: it depends only on $S$. The set $\mathbf{V}(\mathfrak{a})$ (or $\mathfrak{a}$ ) is called the top of $S$, whereas $\mathbf{V}(\mathfrak{b}$ ) (or $\mathfrak{b}$ ) is called the hole of $S$.

Remark 2.2 If $[\mathfrak{a}, \mathfrak{b}]=\operatorname{CREP}(S)$, then $S$ is closed if and only if $\mathfrak{b}=\langle 1\rangle$.

Remark 2.3 Note that if $S$ is empty, then $\mathfrak{a}=\langle 1\rangle, \mathfrak{b}=\langle 1\rangle$.

The following Proposition explains how to obtain $\operatorname{CREP}(S)=[\mathfrak{a}, \mathfrak{b}]$ for a locally closed set $S$ given in the form $S=\mathbf{V}(P) \backslash \mathbf{V}(Q)$ by two ideals $P$ and $Q$. It uses the decomposition of $\mathbf{V}(P)$ into irreducible varieties, which can be done by [8] algorithm.

Proposition 2.4 Let $S=\mathbf{V}(P) \backslash \mathbf{V}(Q)$ be a locally closed set given by two ideals $P$ and $Q$, and let $\left\{\mathfrak{p}_{1}^{\prime}, \ldots, \mathfrak{p}_{s}^{\prime}\right\}$ be the prime decomposition of $P$. Consider the set $\left\{\mathfrak{p}_{1}, \ldots, \mathfrak{p}_{r}\right\}$ of ideals $\mathfrak{p}_{i}^{\prime}$ such that $\mathbf{V}\left(\mathfrak{p}_{i}^{\prime}\right) \nsubseteq \mathbf{V}(Q)$. Then, the $C$-representation $[\mathfrak{a}, \mathfrak{b}]$ of $S$ satisfies

(i) $\mathfrak{a}=\bigcap_{i=1}^{r} \mathfrak{p}_{i}$;

(ii) $\mathfrak{b}=\operatorname{RAD}(\mathfrak{a}+Q)$;

(iii) if $S$ is non-empty then $\mathfrak{a} \subset \mathfrak{b}$.

Proof (i) For $i \in[s]$ let $V_{i}=\mathbf{V}\left(\mathfrak{p}_{i}^{\prime}\right)$. Then $\mathbf{V}(P)=V_{1} \cup \cdots \cup V_{s}$ is the decomposition of $\mathbf{V}(P)$ into irreducible varieties. We have

$S=\mathbf{V}(P) \backslash \mathbf{V}(Q)=\left(\bigcup_{i=1}^{s} V_{i}\right) \backslash \mathbf{V}(Q)=\bigcup_{i=1}^{s}\left(V_{i} \backslash\left(\mathbf{V}(Q) \cap V_{i}\right)\right)$.

Let $J=\left\{i \in[s]: \mathbf{V}\left(\mathfrak{p}_{i}\right) \nsubseteq \mathbf{V}(Q)\right\}$. If $i \in[s] \backslash J$, then $V_{i} \cap \mathbf{V}(Q)=V_{i}$ and so $V_{i} \backslash\left(\mathbf{V}(Q) \cap V_{i}\right)=\emptyset$. Thus the set $V_{i} \backslash\left(\mathbf{V}(Q) \cap V_{i}\right)$ can be excluded from the union, obtaining

$S=\bigcup_{i \in J}\left(V_{i} \backslash\left(\mathbf{V}(Q) \cap V_{i}\right)\right)$.

For $i \in J$, we have $\mathbf{V}(Q) \cap V_{i} \subset V_{i}$. As $V_{i}$ is irreducible, the closure of $V_{i} \backslash\left(\mathbf{V}(Q) \cap V_{i}\right)$ is $V_{i}$. Therefore,

$$
\begin{aligned}
& \bar{S}=\bigcup_{i \in J} V_{i}=\bigcap_{i=1}^{r} \mathbf{V}\left(\mathfrak{p}_{i}\right), \\
& \mathfrak{a}=\mathbf{I}(\bar{S})=\bigcap_{i \in J} \mathbf{I}\left(V_{i}\right)=\bigcap_{i \in J} \mathfrak{p}_{i}^{\prime}=\bigcap_{i=1}^{r} \mathfrak{p}_{i} .
\end{aligned}
$$

(ii) To obtain $\mathfrak{b}=\mathbf{I}(\bar{S} \backslash S)$ note that

$$
\begin{aligned}
& S=\bigcup_{i \in J}\left(V_{i} \backslash\left(\mathbf{V}(Q) \cap V_{i}\right)\right)=\bigcup_{i \in J}\left(V_{i} \backslash \mathbf{V}(Q)\right)=\left(\bigcup_{i \in J} V_{i}\right) \backslash \mathbf{V}(Q)=\bar{S} \backslash V(Q), \\
& \bar{S} \backslash S=\bar{S} \backslash(\bar{S} \backslash \mathbf{V}(Q))=\bar{S} \cap \mathbf{V}(Q)=\mathbf{V}(\mathfrak{a}) \cap \mathbf{V}(Q)=\mathbf{V}(\mathfrak{a}+Q),
\end{aligned}
$$

so that, $\mathfrak{b}=\mathbf{I}(\bar{S} \backslash S)=\operatorname{RAD}(\mathfrak{a}+Q)$.

(iii) From $\mathfrak{b}=\operatorname{RAD}(\mathfrak{a}+Q)$, clearly $\mathfrak{b} \supseteq \mathfrak{a}$. Now $\mathfrak{b}=\mathfrak{a}$ implies $S=\emptyset$ and in this case $\mathfrak{a}=\mathfrak{b}=\langle 1\rangle$. Therefore, if $S$ is non-empty, then $\mathfrak{b} \supset \mathfrak{a}$.

We can further decompose $\operatorname{CREP}(S)=[\mathfrak{a}, \mathfrak{b}]$ and obtain another representation of $S$. Let $\left\{\mathfrak{p}_{i}: i \in[r]\right\}$ be the prime decomposition of $\mathfrak{a}$ and for $i \in[r]$ let $\left\{\mathfrak{p}_{i j}: j \in\left[r_{i}\right]\right\}$ be the prime decomposition of $\mathfrak{p}_{i}+\mathfrak{b}$. The set

$\operatorname{PREP}(S)=\left\{\left[\mathfrak{p}_{i},\left\{\mathfrak{p}_{i j}: j \in\left[r_{i}\right]\right\}\right]: i \in[r]\right\}$ 
is called the P-representation of $S$. Note that it only depends on $S$. Each $\left[\mathfrak{p}_{i},\left\{\mathfrak{p}_{i j}: j \in\left[r_{i}\right]\right\}\right]$ is called a component of $S$, from which $\mathbf{V}\left(\mathfrak{p}_{i}\right)$ (or $\mathfrak{p}_{i}$ ) is the top and $\mathbf{V}\left(\mathfrak{p}_{i j}\right)$ (or $\mathfrak{p}_{i j}$ ) with $j \in\left[r_{i}\right]$ its holes.

Remark 2.5 Note that if $S=\emptyset$, then $\operatorname{PrEP}(S)=\{[\langle 1\rangle,\{\langle 1\rangle\}]\}$.

Proposition 2.6 Let $[\mathfrak{a}, \mathfrak{b}]$ and $\left\{\left[\mathfrak{p}_{i},\left\{\mathfrak{p}_{i j}: j \in\left[r_{i}\right]\right\}\right]: i \in[r]\right\}$ be respectively the $C$-representation and $P$ representation of a locally closed set $S$. Then

(i) If $S \neq \emptyset$, then $\mathfrak{p}_{i} \subset \mathfrak{p}_{i j}$, for all $i \in[r]$ and $j \in\left[r_{i}\right]$;

(ii) $\mathfrak{a}=\bigcap_{i=1}^{r} \mathfrak{p}_{i}$;

(iii) $\mathfrak{b}=\bigcap_{i=1}^{r} \bigcap_{j=1}^{r_{i}} \mathfrak{p}_{i j}$;

(iv) $S=\bigcup_{i=1}^{r}\left(\mathbf{V}\left(\mathfrak{p}_{i}\right) \backslash\left(\bigcup_{j=1}^{r_{i}} \mathbf{V}\left(\mathfrak{p}_{i j}\right)\right)\right)$.

Proof (i) It is consequence of the definition of P-representation, as $\mathfrak{b} \not \subset \mathfrak{p}_{i}$ for non empty $S$.

(ii) It is consequence of Proposition 2.4.

(iii) Considering the intersection of all the identities $\operatorname{RAD}\left(\mathfrak{p}_{\mathfrak{i}}+\mathfrak{b}\right)=\mathfrak{p}_{i_{1}} \cap \cdots \cap \mathfrak{p}_{i_{r_{i}}}$ we have

$$
T=\bigcap_{i=1}^{r} \bigcap_{j=1}^{r_{i}} \mathfrak{p}_{i j}=\bigcap_{i=1}^{r} \operatorname{RAD}\left(\mathfrak{p}_{i}+\mathfrak{b}\right)=\operatorname{RAD}\left(\bigcap_{i=1}^{r}\left(\mathfrak{p}_{i}+\mathfrak{b}\right)\right)
$$

and

$$
\begin{aligned}
\mathbf{V}(T) & =\mathbf{V}\left(\bigcap_{i=1}^{r}\left(\mathfrak{p}_{i}+\mathfrak{b}\right)\right)=\bigcup_{i=1}^{r} \mathbf{V}\left(\mathfrak{p}_{i}+\mathfrak{b}\right)=\bigcup_{i=1}^{r}\left(\mathbf{V}\left(\mathfrak{p}_{i}\right) \cap \mathbf{V}(\mathfrak{b})\right) \\
& =\left(\bigcup_{i=1}^{r} \mathbf{V}\left(\mathfrak{p}_{i}\right)\right) \cap \mathbf{V}(\mathfrak{b})=\mathbf{V}(\mathfrak{a}) \cap \mathbf{V}(\mathfrak{b})=\mathbf{V}(\mathfrak{b}) .
\end{aligned}
$$

Taking ideals of the varieties and using the Nullstellensatz, we have $\mathfrak{b}=T$, so that (iii) is proved.

(iv) As $[\mathfrak{a}, \mathfrak{b}]$ is the C-representation of $S$, we have $S=\mathbf{V}(\mathfrak{a}) \backslash \mathbf{V}(\mathfrak{b})$. Then, by taking varieties in (ii) and (iii), we have

$$
S=\mathbf{V}(\mathfrak{a}) \backslash \mathbf{V}(\mathfrak{b})=\left(\bigcup_{i=1}^{r} \mathbf{V}\left(\mathfrak{p}_{i}\right)\right) \backslash\left(\bigcup_{i=1}^{r} \bigcup_{j=1}^{r_{i}} \mathbf{V}\left(\mathfrak{p}_{i j}\right)\right)=\bigcup_{i=1}^{r}\left(\mathbf{V}\left(\mathfrak{p}_{i}\right) \backslash \bigcup_{j=1}^{r_{i}} \mathbf{V}\left(\mathfrak{p}_{i j}\right)\right)
$$

Proposition 2.7 Let $S$ be a non empty locally closed set with

$\operatorname{CREP}(S)=[\mathfrak{a}, \mathfrak{b}]$ and $\operatorname{PREP}(S)=\left\{\left[\mathfrak{p}_{i},\left\{\mathfrak{p}_{i j}: j \in\left[r_{i}\right]\right\}\right]: i \in[r]\right\}$.

Then

(i) $\operatorname{dim} \mathbf{V}\left(\mathfrak{p}_{i j}\right)<\operatorname{dim} \mathbf{V}\left(\mathfrak{p}_{i}\right)$ for all $i \in[r]$ and $j \in\left[r_{i}\right]$;

(ii) $\operatorname{dim} \mathbf{V}(\mathfrak{b})<\operatorname{dim} \mathbf{V}(\mathfrak{a})$.

Proof (i) As the $\mathfrak{p}_{i}$ and $\mathfrak{p}_{i j}$ are prime and correspond to irreducible varieties the result is obvious.

(ii) From Proposition 2.6 (iii), we have

$$
\begin{aligned}
\operatorname{dim} V(\mathfrak{b}) & =\operatorname{dim} \bigcup_{i=1}^{r} \bigcup_{j=1}^{r_{i}} \mathbf{V}\left(\mathfrak{p}_{i j}\right) \\
& =\max \left\{\operatorname{dim} \mathbf{V}\left(\mathfrak{p}_{i j}\right): i \in[r], j \in\left[r_{i}\right]\right\} \\
& <\max \left\{\operatorname{dim} \mathbf{V}\left(\mathfrak{p}_{i}\right): i \in[r]\right\}=\operatorname{dim} V(\mathfrak{a}) .
\end{aligned}
$$




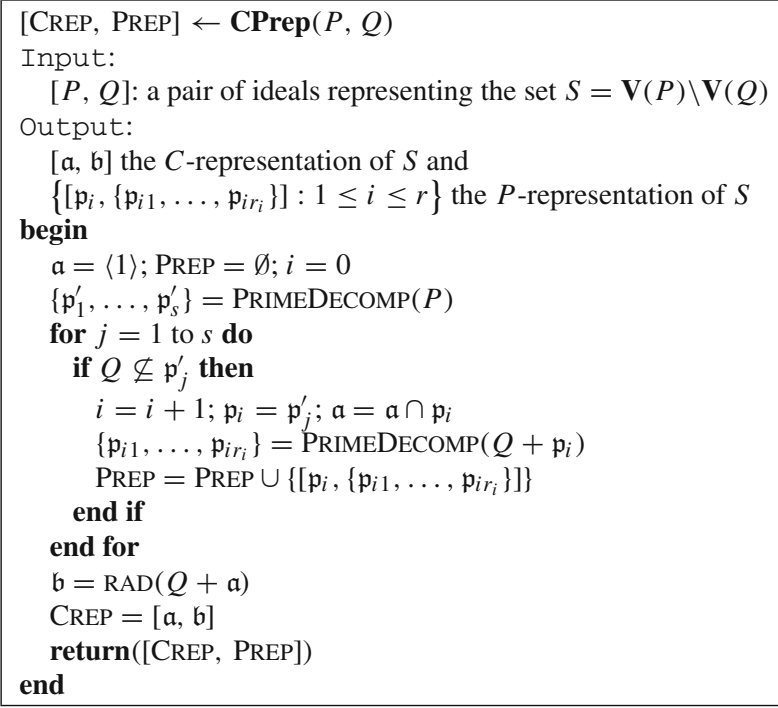

Algorithm 1: CPREP

Corollary 2.8 Let $V$ and $W$ be varieties and $S=V \backslash W$. If $W \subset V$ and $V=\bar{S}$, then $\operatorname{CREP}(S)=[\mathbf{I}(V), \mathbf{I}(W)]$ and $\operatorname{dim} W<\operatorname{dim} V$.

Proof If $\bar{S}=V$ then $\mathfrak{a}=\mathbf{I}(\bar{S})=\mathbf{I}(V)$. Moreover

$\bar{S} \backslash S=\left(\bar{S} \cap\left(\bar{S}^{c} \cup W\right)=\bar{S} \cap W=W\right.$.

Thus $\mathfrak{b}=\mathbf{I}(W)$. The dimension relation is a consequence of Proposition 2.7.

Proposition 2.4 and the definition of P-representation justify Algorithm 1 CPREP for obtaining the canonical representations CREP and PREP of a locally closed set $S=\mathbf{V}(P) \backslash \mathbf{V}(Q)$ given by a pair of ideals $[P, Q]$. The algorithm can be easily modified for obtaining only the $C$-representation $\operatorname{CREP}(S)$ of $S$.

Remark 2.9 If the set $S$ is empty, the algorithm for obtaining $\operatorname{CPREP}(S)$ will return $\operatorname{CPREP}(S)=[[\langle 1\rangle,\langle 1\rangle],\{[\langle 1\rangle$, $\{\langle 1\rangle\}]\}]$.

\section{Canonical Representation of Constructible Sets}

A set $S \subseteq \mathbb{C}^{n}$ is constructible if it is a finite union of locally closed sets. In particular, locally closed sets are constructible. Constructible sets appear naturally in solving parametric polynomial systems of equations. Many authors give special representations for constructible sets $[4,10,12,13]$, adequate for its goals. Our goal is developing the invariant sequence of a constructible set described in [10] setting the outlook on its effective computation, to generalize the CREP of a locally closed set.

Next lemma recalls the behaviour of locally closed sets and constructible sets respect to union, intersection and complementation. We omit the proofs which are straightforward.

\section{Lemma 3.1 .}

(i) If $S$ is locally closed, then $S^{c}$ is constructible;

(ii) If $S_{1}$ and $S_{2}$ are locally closed, then $S_{1} \cup S_{2}$ is constructible and $S_{1} \cap S_{2}$ is locally closed;

(iii) If $S_{1}$ is locally closed and $S_{2}$ is constructible, then $S_{1} \cup S_{2}$ and $S_{1} \cap S_{2}$ are constructible; 
(iv) If $S_{1}$ and $S_{2}$ are constructible, then $S_{1} \cup S_{2}$ and $S_{1} \cap S_{2}$ are constructible.

(v) if $S$ is constructible, then $S^{c}$ is constructible.

(vi) if $S_{1}$ and $S_{2}$ are constructible, then $S_{1} \backslash S_{2}$ is constructible.

In the following $\mathcal{L}$ denotes the family of locally closed sets and $\mathcal{C}$ the family of constructible sets.

Remark 3.2 According to Lemma 3.1, if $S_{1}$ and $S_{2}$ are constructible, then $S_{1} \cup S_{2}, S_{1} \cap S_{2}$ and $S_{1}^{c}$ are constructible sets, too. Then $\mathcal{C}$ is a Boolean algebra of subsets of $\mathbb{C}^{n}$ containing $\mathcal{L}$. On the other hand, if a Boolean algebra $\mathcal{A}$ contains $\mathcal{L}$ then it must contain the finite union of locally closed sets, that is, $\mathcal{C} \subseteq \mathcal{A}$. We conclude that $\mathcal{C}$ is the Boolean algebra generated by $\mathcal{L}$. Let $\mathcal{T}$ be the union of the family of open sets and the family of closed sets. The boolean algebra generated by $\mathcal{T}$ contains $\mathcal{L}$, so $\mathcal{C}$ is also the boolean algebra generated by $\mathcal{T}$.

The first step of the construction of the canonical structure of the constructible set $S$ given as a union of locally closed sets is to separate $\bar{S}$ into two disjoint sets: $\bar{S}=S \uplus C$ where $C$ is the complement of $S$ with respect to $\bar{S}$. Having this in mind we define:

$\mathbf{C}(S)=\bar{S} \backslash S, \quad \mathbf{L}(S)=\bar{S} \backslash \overline{\mathbf{C}(S)}$,

(If the set $S$ is clear from the context, we often write $C$ and $L$ instead of $\mathbf{C}(S)$ and $\mathbf{L}(S)$ respectively).

If $S \in \mathcal{C}$, then, $\bar{S}$ and $S^{c}$ are constructible and $\mathbf{C}(S)=\bar{S} \backslash S$ is a difference of constructibles, so it is a constructible set. Thus, the map

$\mathrm{C}: \mathcal{C} \rightarrow \mathcal{C}$

$S \mapsto \mathbf{C}(S)=\bar{S} \backslash S$

is well defined. Note:

(i) $\bar{S}=\mathbf{C}(S) \uplus S$;

(ii) $S$ is closed if and only if $\mathbf{C}(S)=\emptyset$;

(iii) $S$ is locally closed if and only if $\mathbf{C}(S)$ is closed.

The set $\mathbf{L}(S)=\bar{S} \backslash \bar{C}$ (where $C=\mathbf{C}(S)$ ) is a difference of closed sets, so it is locally closed. Then,

$$
\begin{aligned}
\mathbf{L}: \mathcal{C} & \rightarrow \mathcal{L} \\
S & \mapsto \mathbf{L}(S)=\bar{S} \backslash \bar{C}
\end{aligned}
$$

is a well defined map. Clearly $\bar{S}=\mathbf{L}(S) \uplus \bar{C}$. Moreover, $\mathbf{L}(S) \subseteq S$. Indeed,

$\mathbf{L}(S)=\bar{S} \backslash \bar{C}=\bar{S} \backslash(\overline{\bar{S} \backslash S}) \subseteq \bar{S} \backslash(\bar{S} \backslash S)=S$.

For a constructible set $S$, the set $\mathbf{L}(S)$ can be characterized as the largest locally closed set included in $S$.

We give now a Proposition that determines an explicit expression of $C$ as a union of locally closed sets in terms of the input expression of $S$.

Proposition 3.3 Let $S=S_{1} \cup \cdots \cup S_{r}$ be a constructible set with each $S_{i}$ locally closed. For $i \in[r]$ let $\operatorname{CREP}\left(S_{i}\right)=$ $\left[\mathfrak{a}_{i}, \mathfrak{b}_{i}\right], V_{i}=\mathbf{V}\left(\mathfrak{a}_{i}\right)$ and $W_{i}=\mathbf{V}\left(\mathfrak{b}_{i}\right)$. Then,

$C=\bar{S} \backslash S=\bigcup_{T \subset[r]}\left(\left(\bigcap_{j \in T} V_{j}^{c}\right) \cap\left(\bigcap_{j \notin T} W_{j}\right)\right)$

$=\bigcup_{T \subset[r]}\left(\left(\bigcap_{j \notin T} W_{j}\right) \backslash\left(\bigcup_{j \in T} V_{j}\right)\right)$. 
Proof We have

$$
\begin{aligned}
S & =\left(V_{1} \backslash W_{1}\right) \cup \cdots \cup\left(V_{r} \backslash W_{r}\right)=\left(V_{1} \cap W_{1}^{c}\right) \cup \cdots \cup\left(V_{r} \cap W_{r}^{c}\right) \\
& =\bigcap_{T \subseteq[r]}\left(\left(\bigcup_{j \in T} V_{j}\right) \cup\left(\bigcup_{j \notin T} W_{j}^{c}\right)\right),
\end{aligned}
$$

and thus

$$
S^{c}=\bigcup_{T \subseteq[r]}\left(\left(\bigcap_{j \in T} V_{j}^{c}\right) \cap\left(\bigcap_{j \notin T} W_{j}\right)\right) .
$$

For a subset $T \subseteq[r]$, let

$Z_{T}=\left(\bigcap_{j \in T} V_{j}^{c}\right) \cap\left(\bigcap_{j \notin T} W_{j}\right)$,

so that $S^{c}=\bigcup_{T \subseteq[r]} Z_{T}$. With this notation, the equality to prove is $\bar{S} \backslash S=\bigcup_{T \subset[r]} Z_{T}$. For a set $T \subseteq[r]$ and an index $\ell \in T$ we have

$V_{\ell} \cap Z_{T} \subseteq V_{\ell} \cap \bigcap_{j \in T} V_{j}^{c} \subseteq V_{\ell} \cap V_{\ell}^{c}=\emptyset$,

(in particular, $V_{\ell} \cap Z_{[r]}=\emptyset$ ) and, if $\ell \notin T$, then $W_{\ell} \subset V_{\ell}$ and

$V_{\ell} \cap \bigcap_{j \notin T} W_{j}=\bigcap_{j \notin T} W_{j}$,

and we have $V_{\ell} \cap Z_{T}=Z_{T}$. Therefore, by using the distributive law,
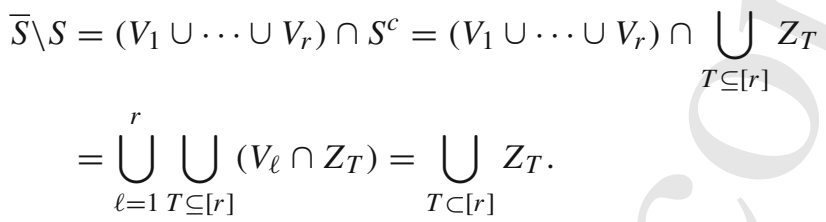

Proposition 3.3 provides an explicit formula of $C=\bar{S} \backslash S$, as a union of locally closed sets. We can compute the CREP of each one of these subsets of $C$ and obtain an expression that allows us to handle $C \subset \bar{S}$ in the same way as we have done with $S$. This provides an iterative method to build the canonical representation of $S$. Next Proposition summarizes the basic properties of the first step in the recursive construction.

Proposition 3.4 Let $S \neq \emptyset$ be a constructible set, $C=\mathbf{C}(S), L=\mathbf{L}(S), \mathfrak{a}=\mathbf{I}(S)$ and $\mathfrak{b}=\mathbf{I}(C)$. Then,

(i) $C \subset \bar{S}$;

(ii) $\bar{C} \subset \bar{S}$;

(iii) $\bar{S}=\bar{L}$;

(iv) $[\mathfrak{a}, \mathfrak{b}]=[\mathbf{I}(S), \mathbf{I}(C)]=[\mathbf{I}(\bar{S})], \mathbf{I}(\bar{C})]$ is the $C$-representation of $L$.

(v) $\operatorname{dim} C<\operatorname{dim} S$. 
Proof (i) Let $S=S_{1} \cup \cdots \cup S_{r}$ with $S_{i}$ locally closed. For $i \in[r]$, let $\operatorname{CREP}\left(S_{i}\right)=\left[\mathfrak{a}_{i}, \mathfrak{b}_{i}\right], V_{i}=\mathbf{V}\left(\mathfrak{a}_{i}\right)$ and $W_{i}=\mathbf{V}\left(\mathfrak{b}_{i}\right)$. Then, $S=\bigcup_{i=1}^{r}\left(V_{i} \backslash W_{i}\right)$ with $W_{i} \subset V_{i}$. By taking closures it results in $\bar{S}=\bigcup_{i=1}^{r} V_{i}$. Now, from formula (3.2) of Proposition 3.3 it results in

$$
C \subseteq \bigcup_{i=1}^{r} W_{i} \subset \bigcup_{i=1}^{r} V_{i}=\bar{S}
$$

(ii) Taking closures in the preceding expression, it results in

$$
\bar{C} \subseteq \bigcup_{i=1}^{r} W_{i} \subset \bigcup_{i=1}^{r} V_{i}=\bar{S} .
$$

(iii) From $\bar{C} \subseteq \bigcup_{j=1}^{r} W_{j}$ we have

$$
L=\bar{S} \backslash \bar{C} \supseteq \bar{S} \backslash\left(\bigcup_{j=1}^{r} W_{j}\right)=\left(\bigcup_{i=1}^{r} V_{i}\right) \backslash\left(\bigcup_{j=1}^{r} W_{j}\right)=\bigcup_{i=1}^{r} \bigcup_{k=1}^{r_{i}}\left(V_{i k} \backslash \bigcup_{j=1}^{r} W_{j}\right),
$$

where $V_{i}=\bigcup_{k=1}^{r_{i}} V_{i k}$ is the decomposition of $V_{i}$ into irreducible varieties. If some irreducible variety $V_{i k}$ of $V_{i}$ of the segment $i$ is cancelled by some $W_{j}$ of a segment $j$, i.e. $W_{j} \supseteq V_{i k}$, then $V_{j} \supset W_{j} \supseteq V_{i k}$, and in this case the variety $V_{i k}$ is included in $V_{j}$. So, $V_{i k}$ does not cancel in the closure of $L$ nor of $S$. Thus $\bar{L} \supseteq \bigcup_{i=1}^{r} V_{i}=\bar{S}$. As $L \subseteq S$ we also have $\bar{L} \subseteq \bar{S}$, and the inclusion is proved.

(iv) and (v) From (ii) and (iii) the expression $L=\bar{S} \backslash \bar{C}$ satisfies the conditions of Corollary 2.8, and thus (iv) and (v) follow.

We proceed now to describe the method for obtaining the canonical representation. Let $S$ be a constructible set. Define the sequence $\left(A_{i}\right)$ by

$A_{1}=S, \quad A_{i+1}=\mathbf{C}\left(A_{i}\right)$.

By Proposition 3.4 (ii) and (v), if $A_{i} \neq \emptyset$, we have $\overline{A_{i}} \supset \overline{A_{i+1}}$ and $\operatorname{dim} \overline{A_{i}}>\operatorname{dim} \overline{A_{i+1}}$. Therefore, there exists an integer $k \geq 1$ such that $A_{k+1}=\emptyset$ and $A_{k}$ is closed. Consider the finite sequences

$S=A_{1}, A_{2}, \ldots, A_{k}, A_{k+1}=\emptyset$

$\bar{S}=\overline{A_{1}} \supset \overline{A_{2}} \supset \cdots \supset \overline{A_{k+1}}=\emptyset$,

$\operatorname{dim}(S)=\operatorname{dim}\left(A_{1}\right)>\operatorname{dim}\left(A_{2}\right)>\cdots>\operatorname{dim}\left(A_{k}\right)$

By construction $A_{2}=\mathbf{C}\left(A_{1}\right)=\bar{S} \backslash S$ is disjoint with $S=A_{1}$. But $A_{3}=\overline{A_{2}} \backslash A_{2}$ is disjoint with $A_{2}$ and a subset of $S$. Thus, we have two decreasing and disjoint subsequences

$$
\begin{aligned}
& S=A_{1} \supset A_{3} \supset \cdots \supset A_{2 \ell \pm 1}, \\
& C=A_{2} \supset A_{4} \supset \cdots \supset A_{2 \ell} .
\end{aligned}
$$

Applying $\mathbf{L}$ to sequence (3.3), i.e. $L_{i}=\overline{A_{i}} \backslash \overline{A_{i+1}}$, we get a new sequence of disjoint sets that fill the whole $\bar{S}$, $L_{1}=\overline{A_{1}} \backslash \overline{A_{2}}, \quad L_{2}=\overline{A_{2}} \backslash \overline{A_{3}}, \ldots, L_{k}=\overline{A_{k}} \backslash \overline{A_{k+1}}=\overline{A_{k}}$

so that

$\bar{S}=\overline{A_{1}}=\overline{A_{1}} \backslash \overline{A_{k+1}}=L_{1} \uplus L_{2} \uplus \cdots \uplus L_{k}$.

As the $L_{i}$ belong alternatively to $S$ and to $C$ the previous sequence is divided into

$$
\begin{aligned}
S & =L_{1} \uplus L_{3} \uplus \cdots \uplus L_{2 \ell \pm 1}, \\
C & =L_{2} \uplus L_{4} \uplus \cdots \uplus L_{2 \ell} .
\end{aligned}
$$




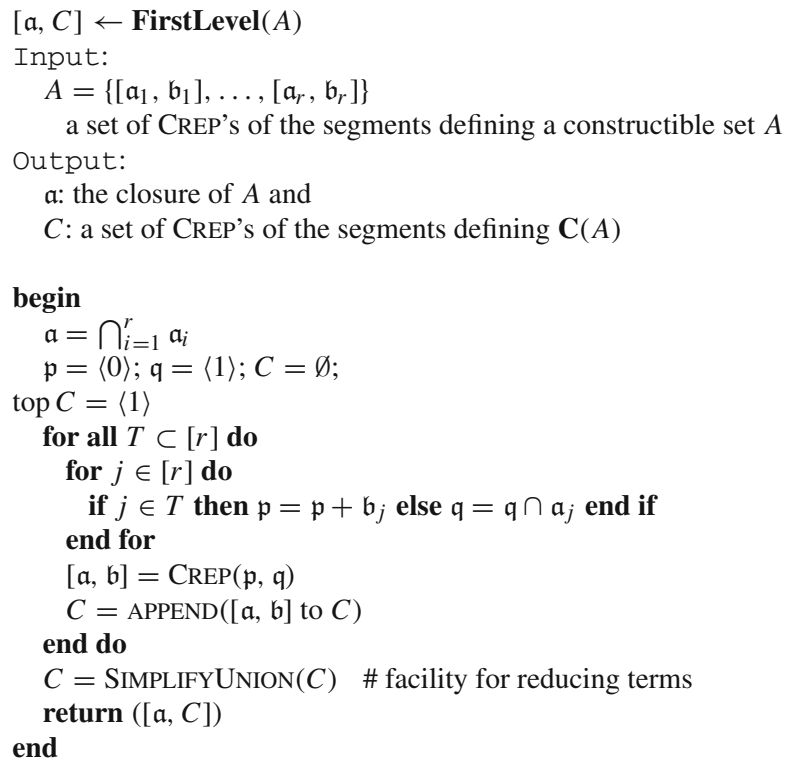

Algorithm 2: FIRSTLEVEL

The odd disjoint locally closed subsets $L_{1}, L_{3} \ldots L_{2 \ell \pm 1}$ in which $S$ is decomposed by the above procedure form the canonical structure of the constructible set $S$ and is independent of the initially given locally closed sets defining $S$. We also obtain the canonical structure of the complement $C=\bar{S} \backslash S$ as the union of the even locally closed subsets $L_{2} \uplus L_{4} \uplus \cdots \uplus L_{2 \ell}$. From them it is obvious how to obtain the canonical representation of $S$ and $C$ whose levels are already given by their CREP's.

For $i \in[k]$, define the ideals $\mathfrak{a}_{i}=\mathbf{I}\left(\overline{A_{i}}\right)$. By using Proposition 3.4 (iv) and (v) it results

$L_{i}=\mathbf{V}\left(\mathfrak{a}_{i}\right) \backslash \mathbf{V}\left(\mathfrak{a}_{i+1}\right)$,

$\operatorname{CREP}\left(L_{i}\right)=\left[\mathfrak{a}_{i}, \mathfrak{a}_{i+1}\right]$,

$\operatorname{dim} \mathbf{V}\left(\mathfrak{a}_{i}\right)>\operatorname{dim} \mathbf{V}\left(\mathfrak{a}_{i+1}\right)$,

$\mathbf{I}(S)=\mathfrak{a}_{1} \subset \mathfrak{a}_{2} \subset \cdots \subset \mathfrak{a}_{k+1}=\langle 1\rangle$,

$\bar{S}=\mathbf{V}\left(\mathfrak{a}_{1}\right) \supset \mathbf{V}\left(\mathfrak{a}_{2}\right) \supset \mathbf{V}\left(\mathfrak{a}_{3}\right) \supset \cdots \supset \mathbf{V}\left(\mathfrak{a}_{k+1}\right)=\emptyset$

Remark 3.5 In $\mathbb{Q}\left[x_{1}, \ldots, x_{n}\right]$, taking into account the decreasing dimensions of the levels of a constructible set we have

(i) The maximum number of levels of $S$ and $C$ is $n+1$, that will occur when $\operatorname{dim}\left(L_{1}\right)=n, \operatorname{dim}\left(L_{2}\right)=n-1, \operatorname{dim}\left(L_{3}\right)=n-2, \ldots, \operatorname{dim}\left(L_{n+1}\right)=0$.

(ii) The maximum number of levels of $S$ is $\left\lfloor\frac{n}{2}\right\rfloor+1$.

(iii) $\operatorname{dim}\left(L_{2 i-1}\right) \geq \operatorname{dim}\left(L_{2 i+1}\right)+2$.

\section{Algorithms for Obtaining the Canonical Representation of a Constructible Set}

The algorithms work with ideals, whereas the definitions of $\mathbf{C}$ and $\mathbf{L}$ as well as the formulas given in the previous sections are given in varieties. To set down the algorithms we must consider the one-to-one correspondence between ideals of varieties and varieties. 


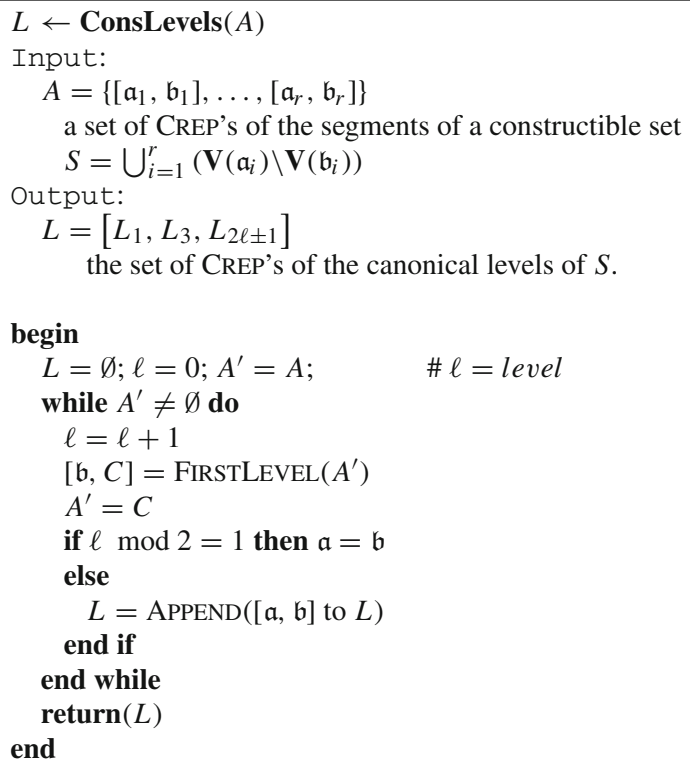

Algorithm 3: CONSLEVELS

To flexibilize the language, if $S=S_{1} \cup \cdots \cup S_{r}$ is a constructible set with each $S_{i}$ locally closed, we call the sets $S_{i}$ the segments of $S$ in the expression $S=S_{1} \cup \cdots \cup S_{r}$.

Algorithm 2 FIRSTLEVEL corresponds to Proposition 3.3. Given a constructible set $S$, we apply the algorithm CREP to its segments; the resulting set of pairs of ideals is the input of FIRSTLEVEL.

FIRSTLEVEL applied to $A_{i}$ returns [a, $A_{i+1}$ ], following Proposition 3.3, where $A_{i+1}=\mathbf{C}\left(A_{i}\right)$ is given by the set of CREP's of its segments, and $\mathfrak{a}$ is the ideal corresponding to the top of $A_{i}$.

FIRSTLEVEL does not return the true level $\mathbf{L}\left(A_{i}\right)$ defined by $A_{i}$ but only its closure. The reason is that the hole will be computed in the next call to FIRSTLEVEL when applied to $A_{i+1}$.

Algorithm 3 CONSLEVEls iterates calls to $\operatorname{FiRSTLEVEL}\left(A_{i}\right)$, obtaining $[\mathfrak{b}, C]$, separating the top $\mathfrak{b}$ and repeating the call with the next $A_{i+1}=C$. But in order to complete the even levels, (i.e. the levels of the constructible), for the odd calls, $\mathfrak{b}$ is reserved setting $\mathfrak{a}=\mathfrak{b}$, whereas for the even calls, the previous level is $L_{i-1}=[\mathfrak{a}, \mathfrak{b}]$. The odd levels $L_{i-1}$ are incorporated to the list $L$ of levels of $S$.

Moreover, the algorithms can be accelerated. Formula (3.2) of Proposition 3.3 for computing the complement $C=\mathbf{C}(S)=\bar{S} \backslash S$ can contain many terms as CREP's of locally closed sets, as it considers all the subsets of $[r]$. Observe that if there are two different segments of $C$ such that $\operatorname{CREP}\left(S_{i}\right)=\left[\mathfrak{a}_{i}, \mathfrak{b}_{i}\right]$ and $\operatorname{CREP}\left(S_{j}\right)=\left[\mathfrak{a}_{j}, \mathfrak{b}_{j}\right]$ are such that $\mathfrak{b}_{i}=\mathfrak{a}_{j}$, then

$S_{i} \cup S_{j}=\left(\mathbf{V}\left(\mathfrak{a}_{i}\right) \backslash \mathbf{V}\left(\mathfrak{b}_{i}\right)\right) \cup\left(\mathbf{V}\left(\mathfrak{b}_{i}\right) \backslash \mathbf{V}\left(\mathfrak{b}_{j}\right)\right)=\mathbf{V}\left(\mathfrak{a}_{i}\right) \backslash \mathbf{V}\left(\mathfrak{b}_{j}\right)$,

so that $\operatorname{CREP}\left(S_{i} \cup S_{j}\right)=\left[\mathfrak{a}_{i}, \mathfrak{b}_{j}\right]$. This can be tested for every $(i, j)$. After this process it can appear more than one segment that has become closed. All of them can be summarized into a single one taking the intersection of the corresponding ideals of varieties. Doing so we can reduce the number of segments in $C$ which will results in an acceleration of the algorithm ConsLEVELS. The acceleration Algorithm 4 SIMPLIFYUNION is to be used inside FIRSTLEVEL after obtaining $C$. Example 3 shows the effectivity of doing so.

In all the algorithms for computing the canonical form of constructible sets we use the CREP of locally closed sets. The reason is that the procedure FIRSTLEVEL uses formulas (3.1) and (3.2) that use CREP, and the iterative procedure CONSLEVELS call it at each step. We remember that in [16], we used PREP for adding together locally closed segments in the algorithm LCUNION, because in this context we know that the considered unions are locally 
closed by Wibmer's Theorem, and so a simpler algorithm can be used. But this is no more applicable for general constructible sets. It is not difficult to transform one representation into the other if we want to compare results.

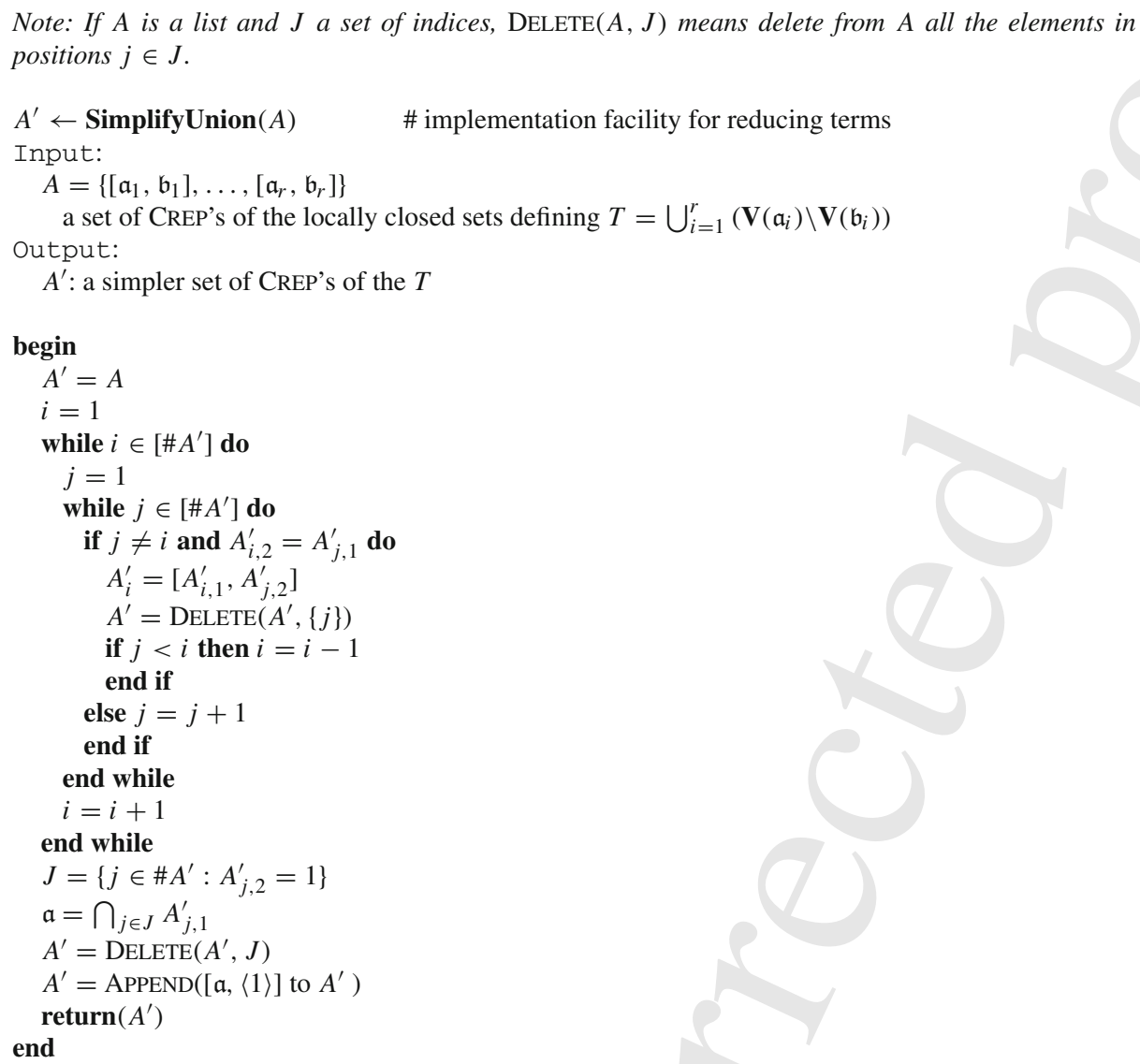

Algorithm 4: SIMPLIFYUNION

\section{Examples}

We have implemented algorithms FIRSTLEVEL and CONSLEVELS (as well as the acceleration routine SIMPLIFYUNION) in Singular. They will be next included in the reformed GROBCOV library. We show here some examples of adding locally closed sets to obtain the canonical representation of the constructible.

Example 1 The first example is a simple geometric problem in 3-dimensional space with a nice geometrical interpretation. Consider the constructible set $S=S_{1} \cup S_{2} \cup S_{3}$, where

$S_{1}=\mathbf{V}\left(x^{2}+y^{2}+z^{2}-1\right) \backslash \mathbf{V}\left(z, x^{2}+y^{2}-1\right)$,

$S_{2}=\mathbf{V}\left(y, x^{2}+z^{2}-1\right) \backslash \mathbf{V}(z(z+1), y, x+z+1)$,

$S_{3}=\mathbf{V}(x) \backslash \mathbf{V}(5 z-4,5 y-3, x)$.

The set $S_{1}$ is a sphere minus a maximum circle, $S_{2}$ is a maximum circle minus two points and $S_{3}$ is a plane minus one point. Applying CONSLEVELS to them the result is: 
Table 1 Segments and bases of Example 2

$S=L_{1} \uplus L_{3}, \quad C=\bar{S} \backslash S=L_{2}$.

$$
\begin{aligned}
& L_{1}=\mathbf{V}\left(x\left(x^{2}+y^{2}+z^{2}-1\right)\right) \backslash \mathbf{V}\left(z, x^{2}+y^{2}-1\right), \\
& L_{2}=\mathbf{V}\left(z, x^{2}+y^{2}-1\right) \backslash \mathbf{V}\left(z, x+y^{2}-1, x y, x^{2}-x\right), \\
& L_{3}=\mathbf{V}\left(z, x+y^{2}-1, x y, x^{2}-x\right) .
\end{aligned}
$$

The canonical representations of $S$ and $C$ are

As expected from the geometrical interpretation, $S_{2}$ is completely included in $S_{1}$ except for the point $P_{1}=$ $\mathbf{V}(z, y, x-1)=\{(1,0,0)\}$. Point $P_{1}$ is not in $S_{1}$ because it is in the circle retrieved from the sphere, and cannot be included in $L_{1}$ because it does not form a locally closed set with $L_{1}$. Thus $S_{1} \cup S_{2}=S_{1} \cup\{(1,0,0)\}$. Now, adding $S_{3}$ will add the plane $x=0$ minus point $(0,3 / 5,4 / 5)$ already contained in $S_{1}$. This implies the addition of the component $\mathbf{V}(x)$, that in order to be included in the first level, from which the maximum circle $\mathbf{V}\left(z, x^{2}+y^{2}-1\right)$ is excluded, will left to be added to the next level the intersection points $P_{2}=(0,1,0)$ and $P_{3}=(-0,-1,0)$. Thus the second level will be $L_{3}=P_{1} \cup P_{2} \cup P_{3}=\{(1,0,0),(0,1,0),(0,-1,0)\}$.

Example 2 We consider now the following system of equations in the context of the computation of its Gröbner Cover [16], in which we can verify the interest of the canonical representation of constructible sets. Consider the ring $R=\mathbb{Q}\left(a_{0}, b_{0}, c_{0}, a_{1}, b_{1}, c_{1}\right)[x, y]$, and the system

$S=\left\{a_{0} x^{2}+b_{0} x+c_{0}, a_{1} x^{2}+b_{1} x+c_{1}\right\}$.

The first step is to compute a CGS (Comprehensive Gröbner System). Using Kapur-Sun-Wang algorithm [11], the parameter space is divided into 11 disjoint segments $S_{1}, \ldots, S_{11}$, and for each segment $S_{i}$ a basis $B_{i}$ specializing 
Table 2 Levels of the canonical representation of Example 2

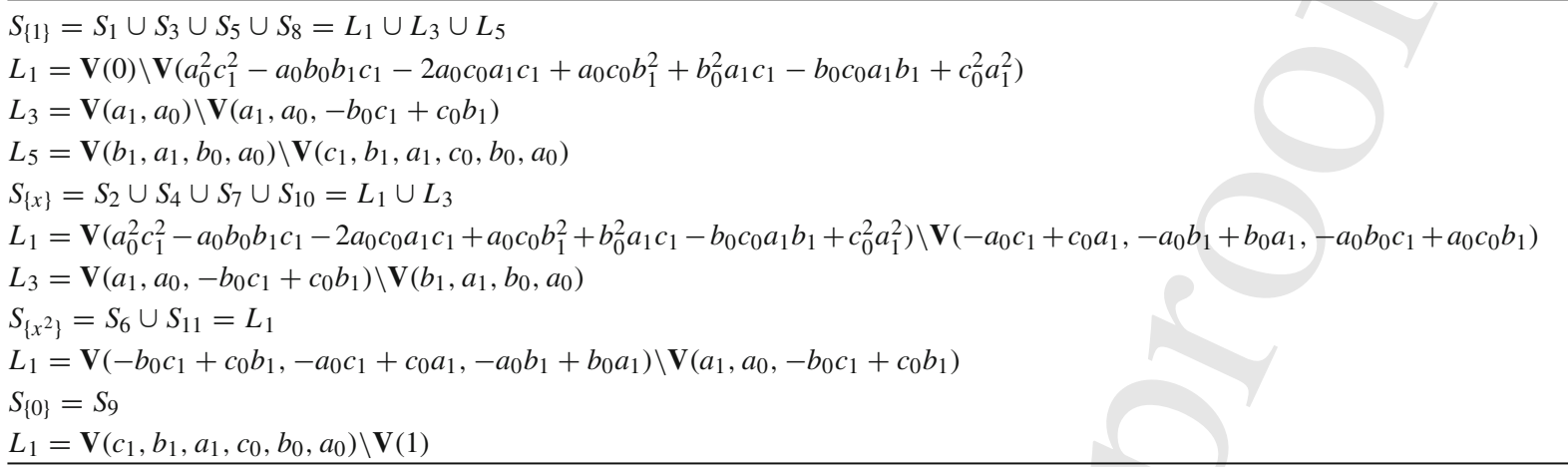

to the reduced Gröbner basis on the whole segment is given. Table 1 gives the sets $S_{i}$ and $B_{i}$. The segments in the CGS are algorithm depending, and can change if we use another algorithm for computing the CGS.

Lets now add together the segments with the same set of lpp's, using CONSLEVELS algorithm. There are four different sets of lpp's (leading power products) in the 11 cases, namely $\{1\},\{x\},\left\{x^{2}\right\}$ and $\{0\}$. We obtain the levels of the canonical representation of the constructible sets formed by the union of the corresponding segments shown in Table 2.

The canonical levels of the constructible sets so obtained do not depend any more on the CGS algorithm used, as each of these segments correspond to a canonical level of all the points of the parameter space with fixed value of the lpp's. We observe that the locally closed segments with fixed lpp's obtained using CONSLEVEL algorithm are identical to the canonical segments of the Gröbner Cover given in CREP representation. Wibmer's Theorem [17], stays that given an homogeneous parametric ideal, the set of points of the parameter space for which the reduced Gröbner basis has a given set of lpp's is parametric (i.e. it accepts a unique reduced Gröbner basis using $I$-regular functions), and is locally closed. So, in general, for the Computation of the Gröbner Cover of non-homogeneous ideals, it is necessary to homogenize the input ideal, then compute its Gröbner Cover and dehomogenize the result. The dehomogenized bases can contain segments with the same sets of lpp. In this example we start with a nonhomogeneous ideal, and instead of homogenizing and using Wibmer's Theorem, we add together the segments of the CGS with fixed lpp of the non-homogeneous ideal using CONSLEVELS (for which Wibmer's Theorem cannot be applied). There is no contradiction in the fact that for this non-homogeneous ideal the sets of points with fixed lpp are not locally closed.

The interesting point that we observe in this example, is that, proceeding in this alternative way, we also recover the canonical segments of the Gröbner Cover. This property will be developed in a next research.

Example 3 To test the effectivity of using the acceleration Algorithm SimPLIFYUniOn, we have applied ConSLEVELS to the output of a CGS containing 26 segments. These segments were grouped into 9 constructible sets by their lpp's. The lpp-sets contained respectively 7, 6, 4, 1, 1, 1, 1, 2, 3 segments. Applying CONSLEVELS to each lpp-segment, each one was reduced to a single segment for each of the 9 constructible sets (i.e. in this example the lpp-segments resulted to be locally closed). We tested times with and without using SIMPLIFYUNION algorithm inside FIRSTLEVEL. The total timing was $7.61 \mathrm{~s}$ using it and $22.07 \mathrm{~s}$ without using it, that justifies the utility of SIMPLIFYUNION.

\section{References}

1. Abanades, M., Botana, F., Montes, A., Recio, T.: An Algebraic taxonomy for locus computation in dynamic geometry. Comput. Aided Geom. D. 56, 22-33 (2014)

2. Adhikari, M.R., Adhikari, A.: Basic modern algebra with applications. Springer, New Delhi (2014) 
3. Allouche, J.P.: Note on the constructible sets of a topological space. In: Papers on general topology and applications (Gorham, ME, 1995). Ann. New York Acad. Sci., vol. 806, pp. 1-10 (1996)

4. Chen, C., Lemaire, F., Li, L., Maza, M.M., Pan, W., Xie, Y.: The Constructiblesettools and parametricsystemtools modules of the regularchains library in maple. In: Proceedings of ICCSA'2008, pp. 342-352. IEEE Computer Society (2008)

5. Cox, D., Little, J., O’Shea, D.: Ideals, varieties and algorithms, vol. 2007, 3rd edn. Springer, New-York (1992)

6. de la Llave, R., Petzold, L., Lorenz, J.: Mathematical robotics: problem 4, two-arm robot. In: Dynamics of algorithms, vol. 118, pp. 1-29. Springer-Verlag (2000)

7. Decker, W., Greuel, G.-M., Pfister, G., Schönemann, H.: Singular 4-0-2. A computer algebra system for polynomial computations. http://www.singular.uni-kl.de

8. Gianni, P., Trager, B., Zacharias, G.: Gröbner bases and primary decomposition of polynomial ideals. J. Symbolic Comput. 6, 149$167(1988)$

9. Gonzalez, M.J., Recio, T.: The ROMIN inverse geometric model and the dynamic evaluation method. In: Computer algebra in industry, pp. 117-141. Wiley (1993)

10. O'Halloran, J., Schilmoeller, M.: Gröbner bases for constructible sets. Commun. Algebra 30(11), 5479-5483 (2002)

11. Kapur, D., Sun, Y., Wang, D.: A new algorithm for computing comprehensive Gröbner systems. In: Proceedings of ISSAC'2010, pp. 29-36. ACM Press, New York (2010)

12. Kemper, G.: Morphisms and constructible sets: making two theorems of Chevalley constructive. Preprint Technische Universität München (2007)

13. Leykin, A.: Constructibility of the set of polynomials with a fixed bernstein-sato polynomial: an algorithmic approach. J. Symbolic Comput. 32(6), 663-675 (2001)

14. Montes, A.: Personal web page. http://www-ma2.upc.edu/ montes

15. Montes, A., Recio, T.: Generalizing the Steiner-Lehmus theorem using the Gröbner cover. Math. Comput. Simul. 104, 67-81 (2014)

16. Montes, A., Wibmer, M.: Gröbner bases for polynomial systems with parameters. J. Symbolic Comput. 45, 1391-1425 (2010)

17. Wibmer, M.: Gröbner bases for families of affine or projective schemes. J. Symbolic Comput. 42, 803-834 (2007) 